\title{
One-dimensional ordering of ultra-low-density ion beams in a storage ring
}

\author{
H. Okamoto, K. Okabe, and Y. Yuri \\ Graduate School of Advanced Sciences of Matter, Hiroshima University, 1-3-1 Kagamiyama, Higashi-Hiroshima 739-8530, Japan \\ D. Möhl \\ CERN, CH-1211 Geneva, Switzerland
}

A. M. Sessler

Lawrence Berkeley National Laboratory, 1 Cyclotron Road, Berkeley, California 94720, USA

(Received 9 October 2003; revised manuscript received 16 April 2004; published 23 June 2004)

\begin{abstract}
The two-particle model, first introduced by Hasse, is employed to predict the beam temperature at which a one-dimensional ordered state of ions will be established in a cooler storage ring. The proposed state does not have the ions (in the beam frame) at rest, but simply has them not passing each other; i.e., remaining in the same (ordered) sequence. The model is applicable to an ultra-low-density beam where collective Coulomb interactions are negligible. It is pointed out that the nature of the anomalous beam behavior observed in electron-cooling experiments at GSI (Darmstadt) and MSL (Stockholm) is approximately free from such parameters as the lattice design, ion species, beam density, and energy. On the basis of the model, which is put in Hamiltonian form, scaled, and numerically studied, a universal criterion of one-dimensional beam ordering at low line density is derived. Analytic work is employed to explain the numerical results and derives an approximate criterion.
\end{abstract}

DOI: 10.1103/PhysRevE.69.066504

PACS number(s): 29.20.Dh, 29.27.Fh, 41.75.-i

\section{INTRODUCTION}

Over twenty years ago, Russian researchers reported on an anomalous behavior of electron-cooled proton beams circulating in the NAP-M storage ring [1]. In order to explain the experimental observation, the concept of a phase transition of a fast stored beam was introduced and discussed. Molecular dynamics (MD) simulations performed to reproduce the NAP-M result [2] actually show that the beam may have been composed of a chain of thin transverse "disks" drifting back and forth in the longitudinal direction.

Steck and co-workers [3] recently observed a similar anomaly of highly charged heavy ion beams in the Experimental Storage Ring (ESR) at GSI; a sudden jump of the momentum spread was detected with an electron cooler turned on, once a certain line density was reached. This curious phenomenon of low-intensity cold beams was reconfirmed later by Danared et al. at CRYRING in Sweden [4]. In either case, the experimental data were interpreted as the possible signature of a longitudinal beam ordering, which was theoretically explained by Hasse through systematic Monte Carlo calculations on a two-particle model [5]. According to his theory, what had been established in these storage rings is a one-dimensional (1D) stringlike order; namely, individual ions still execute large transverse oscillations but no longer pass each other longitudinally. He then explored the collective motion of a Coulomb string to describe the fundamental mechanism behind the ESR and CRYRING observations [6].

In this paper, we make a step forward from the Hasse's numerical approach in Ref. [5] to give a simple and universal criterion of $1 \mathrm{D}$ ordering. The present model is valid for small linear density and has thus, to first order, nothing to do with Coulomb crystals and their stability where the collective space-charge interactions play a definitive role [6-8]. This assumption seems quite reasonable for the analysis of the present experiments, considering that the jump of the momentum spread takes place when the total number of stored ions reaches on the order of 1000 at the ESR, i.e., at an average interparticle distance of about $10 \mathrm{~cm}$. In such an ultra-low-density regime, no collective effects could dominate the beam. We thus assume that, even after the transition, the motions of individual ions are roughly independent of each other [9]. The orbit of the $i$ th ion then obeys the following single-particle Hamiltonian in the absence of collisions:

$$
\begin{aligned}
H_{i}= & \frac{1}{2}\left[\left(p_{x}^{(i)}\right)^{2}+\left(p_{y}^{(i)}\right)^{2}+\left(p_{z}^{(i)}\right)^{2}\right]-\frac{\gamma}{\rho} x^{(i)} p_{z}^{(i)} \\
& +\frac{1}{2}\left[K_{x}(s)\left(x^{(i)}\right)^{2}+K_{y}(s)\left(y^{(i)}\right)^{2}\right],
\end{aligned}
$$

where the canonical coordinates $\left(x^{(i)}, y^{(i)}, z^{(i)} ; p_{x}^{(i)}, p_{y}^{(i)}, p_{z}^{(i)}\right)$ have been defined in the rest frame, $K_{x}$ and $K_{y}$ are the focusing functions determined by the arrangement of quadrupole magnets, $\rho$ is the local curvature of the design beam orbit, $\gamma$ is the Lorentz factor, and we have taken the path length $s$ as the independent variable. Since the degrees of freedom are only weakly coupled (even when particle interaction is included), it is not surprising that the beam maintains a large temperature anisotropy between the transverse and longitudinal directions. It is also worthy to emphasize that our model does not explicitly depend on the line density of the beam. The line density itself, in other words the average distance between neighboring ions, appears to be inessential in this unique phenomenon. The critical density is most 
likely determined by the ability of the cooling system rather than by the physics of "collective" ion interactions. The anomalous Schottky signals were, indeed, observed at a certain density, but that should be due to the density dependence of the equilibrium temperature of an electron-cooled beam [10]. We thus speculate that the beam temperature achievable with a specific cooler is most important.

The paper is organized as follows: In Sec. II, we briefly review the current understandings of the ESR-type event to elucidate the purpose and motivation of this work. The twoparticle model relevant to the $1 \mathrm{D}$ ordering effect is then developed in Sec. III. Our model is adequate only when the line density of a beam is low (just as in the ESR and CRYRING situations). Although the basic approach is similar to Hasse's in Ref. [5], we here provide a formalism that enables us to clarify the underlying physics and to establish a universal criterion. In fact, under some simplifying assumptions, the dynamical system turns out to be substantially free from any parameters. In Sec. IV, results of systematic two-particle simulations are given to identify the operating region where the ordered state will be realized. On the basis of the universal Hamiltonian, we derive, in Sec. V, a very simple criterion for the transition temperature. This work provides an intuitive description of the physical process behind the transition. Finally, the results are summarized in Sec. VI.

\section{PREVIOUS MODELS}

The ESR observation of ordering resembles the famous NAP-M event from which the concept of beam crystallization was born [11]. To our knowledge, there are two separate theories developed for the $1 \mathrm{D}$ ordering effect $[5,6,12]$. As mentioned already, Hasse performed Monte Carlo simulations based on a "two-particle model," successfully reproducing the ESR outputs [5]. The model predicts the temperature below which ordering occurs, but not the density below which ordering can be achieved. A collective theory was constructed later by him, to predict the linear density and beam temperature at which the anomaly shows up [6]. Examining the excitation and stability of the collective "zigzag" mode, Hasse eventually proposed four criteria of 1D beam ordering:

$$
\begin{gathered}
\lambda<0.709, \quad \frac{\tau_{\|}}{\tau_{\beta}}>\frac{1}{\lambda}, \\
k_{B} T_{\perp}<0.25 \frac{q^{2}}{4 \pi \varepsilon_{0} a_{W S}}, \\
k_{B} T_{\|}<0.7 \frac{q^{2}}{4 \pi \varepsilon_{0} a_{W S}},
\end{gathered}
$$

where $q$ is the charge state of ions, $k_{B}$ is the Boltzmann constant, $T_{\perp}$ and $T_{\|}$are the transverse and longitudinal beam temperature whose careful definition appears in Eq. (12) below, $\tau_{\|}$and $\tau_{\beta}$ are the period of an average Coulomb scattering and that of a single betatron oscillation, $a_{W S}$ is the Wigner-Seitz radius, and $\lambda$ is the density parameter defined by $\lambda=a_{W S} / \bar{d}$ with $\bar{d}$ being the average interparticle distance
[6]. The Wigner-Seitz radius is determined by $4 \pi a_{W S}^{3} / 3$ $=1 / \rho_{v}$, where $\rho_{v}$ is the density of particles in a threedimensional beam and under the same focusing conditions, but in the limit of many particles. At low temperature limit, we have $a_{W S}=\left(3 q^{2} / 8 \pi \varepsilon_{0} m \omega_{\beta}^{2}\right)^{1 / 3}$ where $m$ is the rest mass of the particle and $\omega_{\beta}$ is the betatron frequency. By defining the longitudinal plasma parameter as $\Gamma_{\|}=\left(q^{2} / 4 \pi \varepsilon_{0} \bar{d}\right) / k_{B} T_{\|}$, the last formula in Eqs. (2) can be simplified to $\Gamma_{\|}>1.43 \lambda$. There is, however, the choice of "distance" in the evaluation of Coulomb energy, and different values of $\Gamma$ (see below for two more) correspond to different choices for this distance.

Meshkov et al. have presented an analytic theory in Ref. [12] somewhat different from the approach of Hasse. Linking the ESR event to beam crystallization, they pointed out the very small $\Gamma_{\|}$at the transition density. In fact, the measured value of $\Gamma_{\|}$is far below the theoretically predicted threshold of the transition to a liquid phase. To explain this discrepancy, they investigated a binary Coulomb collision and concluded two criteria. Their first criterion can be expressed as [12]

$$
\Gamma_{1} \equiv \frac{q^{2}}{4 \pi \varepsilon_{0} \bar{d}} \frac{1}{k_{B} \sqrt{T_{\perp} T_{\|}}}<1
$$

It can be shown, however, that this criterion is not generally required. We can construct cases where $\Gamma_{1}>1$ and yet $1 \mathrm{D}$ ordering (as verified, e.g., by the code described below) occurs. In any event the condition (3) can hardly be broken in the cases of interest to us here because of a very large $\bar{d}$; as can be concluded from Table 1 of Ref. [12] where $\Gamma_{1}$ is shown to be of the order of $10^{-3}-10^{-5}$ for the present experiments.

On the other hand, by requiring that two colliding particles move longitudinally, during half a betatron period, by less than the maximum impact parameter still leading to reflection, a second criterion was derived [12]:

$$
\Gamma_{2} \equiv \frac{q^{2}}{4 \pi \varepsilon_{0} a} \frac{1}{k_{B} T_{\|}}>\pi,
$$

where $a$ denotes the transverse beam size. Since $a \propto \sqrt{T_{\perp}}$, the criterion (4) has the temperature-dependence

$$
T_{\perp} T_{\|}^{2}<\text { const. }
$$

This is consistent with one of our results below (Sec. V).

Now, we can raise many questions, recalling the fact that the heavy-ion beams in ESR and CRYRING were all in the ultra-low density regime at transition: Can the ESR-type event really be linked to the concept of a phase transition in the beam? Do the physical quantities like the Wigner-Seitz radius, $\Gamma$ parameters, beam density, and so on, play an important role in this phenomenon? Is it really possible to see collective modes excited even when individual ions are roughly $10 \mathrm{~cm}$ apart? In the following sections, we try to answer these questions. 


\section{UNIVERSAL APPROACH}

\section{A. Two-particle Hamiltonian}

Suppose an ultra-low-density ordered beam in a storage ring; each particle is simply longitudinally reflected back at every collision with its neighbors. We then reduce the number of ions, so that the average interparticle distance becomes larger and larger. Clearly, the ordered state can be maintained during this process. Note that we can make the plasma parameter arbitrarily small by removing particles (provided that the temperature change is negligible). Such an ordered system may not be categorized as a conventional liquid state where the $\Gamma$ parameter is supposed to be on the order of 1 or even greater.

It is quite natural to say that collective effects are negligible at ultra-low density. The Coulomb interaction occurs only between two neighboring ions that happen to come close to each other. The Hamiltonian governing this binary process is given, in the rest frame, by

$$
H=H_{1}+H_{2}+\frac{r_{p}}{\beta^{2} \gamma^{2}} \frac{1}{d},
$$

where the classical radius of the ion is $r_{p} \equiv q^{2} / 4 \pi \varepsilon_{0} m c^{2}$ with $c$ being the speed of light, $d$ is the interparticle distance, $H_{i}(i=1,2)$ is the single-particle Hamiltonian already introduced in Eq. (1), $\beta=\sqrt{1-1 / \gamma^{2}}$, and the last term describes the Coulomb interaction. Since the collision rate is extremely low due to the large average interparticle distance and the low longitudinal temperature, each ion executes many betatron oscillations during the time interval from one collision to the next. This suggests that the details of the lattice structure are not very important. We thus employ the smooth approximation, replacing $K_{x}$ and $K_{y}$, respectively, by $\left(\nu_{x} / R\right)^{2}$ and $\left(\nu_{y} / R\right)^{2}$ where $\nu_{x(y)}$ is the betatron tune, and $R$ denotes the average radius of the ring. For the sake of simplicity, we neglect the effect of bending magnets that is also believed to be unimportant in the present case.

In the following, we will mainly discuss the situation of nearly equal betatron tunes, i.e., $\nu_{x} \approx \nu_{y}$, as was the case in the ESR and CRYRING experiments [13]. We have actually confirmed, through a number of simulations, that the influence of unequal tunes is weak and the results depend very little on tune ratio $\nu_{x} / \nu_{y}$. Only in "pathological cases" where $\nu_{x} / \nu_{y}$ is quite different from unity and the transverse temperature exceedingly large $\left(T_{\perp} \gg 100 T_{\|}\right)$, is a difference in transition temperature noticeable. With $\nu_{x} \approx \nu_{y}\left(\equiv \nu_{0}\right)$, Eq. (6) can be written as

$$
\begin{aligned}
H= & \sum_{i=1,2}\left\{\frac{\left(p_{x}^{(i)}\right)^{2}+\left(p_{y}^{(i)}\right)^{2}+\left(p_{z}^{(i)}\right)^{2}}{2}+\frac{1}{2}\left(\frac{\nu_{0}}{R}\right)^{2}\left[\left(x^{(i)}\right)^{2}+\left(y^{(i)}\right)^{2}\right]\right\} \\
& +\frac{r_{p}}{\beta^{2} \gamma^{2}} \frac{1}{\sqrt{\left(x^{(1)}-x^{(2)}\right)^{2}+\left(y^{(1)}-y^{(2)}\right)^{2}+\left(z^{(1)}-z^{(2)}\right)^{2}}} .
\end{aligned}
$$

It is easy to derive the momentum conservation law $p_{z}^{(1)}$ $+p_{z}^{(2)}=$ const from the Hamiltonian (7). Without loss of generality, we assume that $p_{z}^{(1)}+p_{z}^{(2)}=0$. The longitudinal equation of relative motion then becomes

$$
\begin{aligned}
& \frac{d^{2}}{d s^{2}}\left(z^{(1)}-z^{(2)}\right) \\
& \quad=\frac{2 r_{p}}{\beta^{2} \gamma^{2}} \frac{z^{(1)}-z^{(2)}}{\left[\left(x^{(1)}-x^{(2)}\right)^{2}+\left(y^{(1)}-y^{(2)}\right)^{2}+\left(z^{(1)}-z^{(2)}\right)^{2}\right]^{3 / 2}} .
\end{aligned}
$$

In the horizontal direction, we have

$$
\begin{aligned}
\frac{d^{2}}{d s^{2}}\left(x^{(1)}-x^{(2)}\right) & \\
= & -\left(\frac{\nu_{0}}{R}\right)^{2}\left(x^{(1)}-x^{(2)}\right) \\
& +\frac{2 r_{p}}{\beta^{2} \gamma^{2}} \frac{x^{(1)}-x^{(2)}}{\left[\left(x^{(1)}-x^{(2)}\right)^{2}+\left(y^{(1)}-y^{(2)}\right)^{2}+\left(z^{(1)}-z^{(2)}\right)^{2}\right]^{3 / 2}},
\end{aligned}
$$

and the similar equation of motion holds in the vertical degree of freedom. Putting $X=x^{(1)}-x^{(2)}, Y=y^{(1)}-y^{(2)}$ and $Z$ $=z^{(1)}-z^{(2)}$, we readily find that these equations of motion are derivable from the Hamiltonian

$$
\begin{aligned}
\tilde{H}= & \frac{1}{2}\left(P_{x}^{2}+P_{y}^{2}+P_{z}^{2}\right)+\frac{1}{2}\left(\frac{\nu_{0}}{R}\right)^{2}\left(X^{2}+Y^{2}\right) \\
& +\frac{2 r_{p}}{\beta^{2} \gamma^{2}} \frac{1}{\sqrt{X^{2}+Y^{2}+Z^{2}}} .
\end{aligned}
$$

When the two colliding particles are far away from each other, the relative motion is a simple drift in the longitudinal direction and a harmonic oscillation in the transverse direction. This is always true for low density, no matter how large the average interparticle distance is (there is a lower but no upper limit for $\bar{d}$ !). The line density of the beam therefore does not play an essential role in our two-particle model.

\section{B. Scaling}

The Hamiltonian (10) is clearly a constant of motion as it does not explicitly depend on $s$. The magnitude of $\widetilde{H}$ averaged over all stored particles is equal to the sum of the energies of three independent motions (the two transverse harmonic oscillations and longitudinal drift) because of the very low line density:

$$
\begin{aligned}
E \equiv\langle\tilde{H}\rangle \approx & \left\langle\frac{P_{x}^{2}}{2}+\frac{1}{2}\left(\frac{\nu_{0}}{R}\right)^{2} X^{2}\right\rangle \\
& +\left\langle\frac{P_{y}^{2}}{2}+\frac{1}{2}\left(\frac{\nu_{0}}{R}\right)^{2} Y^{2}\right\rangle+\left\langle\frac{P_{z}^{2}}{2}\right\rangle,
\end{aligned}
$$

where $\langle A\rangle$ stands for the mean value of the quantity $A$. Noting that $\left\langle X^{2}\right\rangle=2\left\langle\left(x^{(1)}\right)^{2}\right\rangle=2\left\langle\left(x^{(2)}\right)^{2}\right\rangle$, etc., we have $E=\left\langle p_{x}^{2}\right\rangle$ $+\left\langle p_{y}^{2}\right\rangle+\left\langle p_{z}^{2}\right\rangle+\left(\nu_{0} / R\right)^{2}\left(\left\langle x^{2}\right\rangle+\left\langle y^{2}\right\rangle\right)$ where the superscript $(i)$ has been dropped for brevity. The longitudinal variable $p_{z}$ can be related to the momentum spread $\delta p / p$ of the beam, measured in the laboratory frame, as $p_{z}=(\delta p / p) / \gamma$. In addition, the principle of energy equipartition requires $\left\langle p_{x}^{2}\right\rangle$ $=\left(\nu_{0} / R\right)^{2}\left\langle x^{2}\right\rangle$ and $\left\langle p_{y}^{2}\right\rangle=\left(\nu_{0} / R\right)^{2}\left\langle y^{2}\right\rangle$. We thus conclude $E$ $=2\left(\left\langle p_{x}^{2}\right\rangle+\left\langle p_{y}^{2}\right\rangle\right)+\left\langle(\delta p / p)^{2}\right\rangle / \gamma^{2}$. Following the useful defini- 
tion of beam temperature (see Steck et al. [3]), we define $T_{\perp}$ and $T_{\|}$as

$$
\begin{gathered}
k_{B} T_{\perp}=\frac{1}{2} m c^{2} \beta^{2} \gamma^{2}\left(\left\langle p_{x}^{2}\right\rangle+\left\langle p_{y}^{2}\right\rangle\right), \\
k_{B} T_{\|}=m c^{2} \beta^{2}\left\langle\left(\frac{\delta p}{p}\right)^{2}\right\rangle .
\end{gathered}
$$

Use of these definitions yields

$$
E \approx \frac{k_{B}}{m(\beta \gamma c)^{2}}\left(4 T_{\perp}+T_{\|}\right) .
$$

Before proceeding to numerical results, we demonstrate that the dynamical system of Eq. (10) is basically parameter free. All we must do for this purpose is to introduce the scaling transformation

$$
\begin{aligned}
& X=\left[\frac{2 r_{p}}{\beta^{2} \gamma^{2}}\left(\frac{R}{\nu_{0}}\right)^{2}\right]^{1 / 3} \hat{x}, \quad P_{x}=\left(\frac{2 r_{p}}{\beta^{2} \gamma^{2}} \frac{\nu_{0}}{R}\right)^{1 / 3} \hat{p}_{x}, \\
& Y=\left[\frac{2 r_{p}}{\beta^{2} \gamma^{2}}\left(\frac{R}{\nu_{0}}\right)^{2}\right]^{1 / 3} \hat{y}, \quad P_{y}=\left(\frac{2 r_{p}}{\beta^{2} \gamma^{2}} \frac{\nu_{0}}{R}\right)^{1 / 3} \hat{p}_{y}, \\
& Z=\left[\frac{2 r_{p}}{\beta^{2} \gamma^{2}}\left(\frac{R}{\nu_{0}}\right)^{2}\right]^{1 / 3} \hat{z}, \quad P_{z}=\left(\frac{2 r_{p}}{\beta^{2} \gamma^{2}} \frac{\nu_{0}}{R}\right)^{1 / 3} \hat{p}_{z} .
\end{aligned}
$$

The Hamiltonian (10) can then be rewritten, with the new variables $\left(\hat{x}, \hat{y}, \hat{z} ; \hat{p}_{x}, \hat{p}_{y}, \hat{p}_{z}\right)$ as

$$
\hat{H}=\frac{1}{2}\left(\hat{p}_{x}^{2}+\hat{p}_{y}^{2}+\hat{p}_{z}^{2}\right)+\frac{1}{2}\left(\hat{x}^{2}+\hat{y}^{2}\right)+\frac{1}{\sqrt{\hat{x}^{2}+\hat{y}^{2}+\hat{z}^{2}}},
$$

where the independent variable has also been scaled to be $\theta=\nu_{0} \cdot(s / R)$. The scaled temperature, defined by $\hat{T}_{\perp} \equiv\left(\left\langle\hat{p}_{x}^{2}\right\rangle\right.$ $\left.+\left\langle\hat{p}_{y}^{2}\right\rangle\right) / 2$ and $\hat{T}_{\|} \equiv\left\langle\hat{p}_{z}^{2}\right\rangle$, can be calculated from

$$
\left\{\begin{array}{c}
\hat{T}_{\perp} \\
\hat{T}_{\|}
\end{array}\right\}=\frac{2 k_{B}}{m c^{2}}\left(2 r_{p} \beta \gamma \frac{\nu_{0}}{R}\right)^{-2 / 3}\left\{\begin{array}{c}
T_{\perp} \\
T_{\|}
\end{array}\right\} .
$$

A different definition of dimensionless temperature has been taken in Ref. [5] where the scaling constant explicitly contains the average interparticle distance. Once such a definition is adopted, the corresponding numerical results naturally depend on the line density of the beam. Certainly, one can obtain dimensionless parameters that way, but the introduction of line density, or equivalently, average particle spacing, is not physically meaningful. By contrast, we have directly scaled the Hamiltonian itself, making it parameter free, and since our scaling constants are totally independent of $\bar{d}$, the present theory cannot produce density-dependent results. The ingredients in all that follow are the scaled Hamiltonian (14) and temperatures (15). They permit us to establish "universal" ordering conditions.

\section{TRANSITION TEMPERATURE}

In order to explore the condition under which the transition to the ordered state is anticipated, we numerically integrated the canonical equations of motion derived from the universal Hamiltonian (14). We are simply investigating the dynamics of two particles. The numerical procedure is as follows:

(i) Choose the longitudinal scaled temperature $\hat{T}_{\|}$and the transverse scaled temperatures $\hat{T}_{x}\left(\equiv\left\langle\hat{p}_{x}^{2}\right\rangle / 2\right) \quad$ and $\hat{T}_{y}\left(\equiv\left\langle\hat{p}_{y}^{2}\right\rangle / 2\right)$.

(ii) Choose an initial value of $\hat{p}_{z}$ arbitrarily from Gaussian random numbers whose standard deviation is equal to $\sqrt{\hat{T}_{\|}}\left(=\sqrt{\left\langle\hat{p}_{z}^{2}\right\rangle}\right.$ ); note that, when the initial $\hat{p}_{z}$ is positive (negative), then the corresponding longitudinal coordinate must initially be negative (positive) so that the two particles eventually collide.

(iii) Choose initial values of the transverse canonical coordinates $\left(\hat{x}, \hat{y} ; \hat{p}_{x}, \hat{p}_{y}\right)$ arbitrarily from two sets of Gaussian random numbers whose standard deviations are equal to $\sqrt{2 \hat{T}_{x}}$ for $\left(\hat{x}, \hat{p}_{x}\right)$ and to $\sqrt{2 \hat{T}_{y}}$ for $\left(\hat{y}, \hat{p}_{y}\right)$.

(iv) As the initial value of $|\hat{z}|$, choose any number much greater than 10 .

(v) Using these initial conditions, numerically solve the canonical equations by means of the symplectic integrator. No information except for the scaled temperature is required for this simulation. We repeat the above procedure several hundred times at fixed temperature $\hat{T}_{\perp}$ and $\hat{T}_{\|}$in order to evaluate the reflection probability, namely, the rate of events where two longitudinally approaching particles are reflected back in a single collision.

Since the present model is relevant only to an ultra-lowdensity beam, it is essential to set the initial value of $|\hat{z}|$ sufficiently large so that the last term in Eq. (14) can be ignored at the beginning. In fact, the two-particle model is based on the assumption that each individual particle "sees" only the nearest neighbor. If the Coulomb potential is not negligible when $|\hat{z}|$ is comparable to the scaled interparticle distance $\widetilde{d} \equiv \bar{d}\left[\left(2 r_{p} / \beta^{2} \gamma^{2}\right)\left(R^{2} / \nu_{0}^{2}\right)\right]^{-1 / 3}$, we must take into account the contribution from, at least, two neighbors at both sides. To know the starting condition appropriate for twoparticle simulations, we performed test runs changing the initial value of $\hat{z}$. Examples are given in Fig. 1 where a round beam $\left(\left\langle\hat{x}^{2}\right\rangle=\left\langle\hat{y}^{2}\right\rangle, \hat{T}_{x}=\hat{T}_{y}\right)$ and two different combinations of $\left(\hat{T}_{\perp}, \hat{T}_{\|}\right)$have been assumed. The ordinate is the reflection probability while the abscissa indicates the initial $\hat{z}$. These pictures point out that the initial value of $|\hat{z}|$ must be chosen well above 10; then, our model produces proper results roughly independent of the line density of the beam. Assuming ${ }^{197} \mathrm{Au}^{79+}$ ions at $360 \mathrm{MeV} / \mathrm{u}$ in ESR as an example, we find that the scaled distance of 100 corresponds to $\bar{d}$ $\approx 1.8 \mathrm{~mm}$ and $\lambda \approx 0.011$.

Figure 2 shows the reflection probability predicted by our model. The abscissa and ordinate represent the scaled temperature $\hat{T}_{\|}$and $\hat{T}_{\perp}$, respectively. The reflection rate at each mesh point on the $\hat{T}_{\|}-\hat{T}_{\perp}$ plane has been evaluated from 

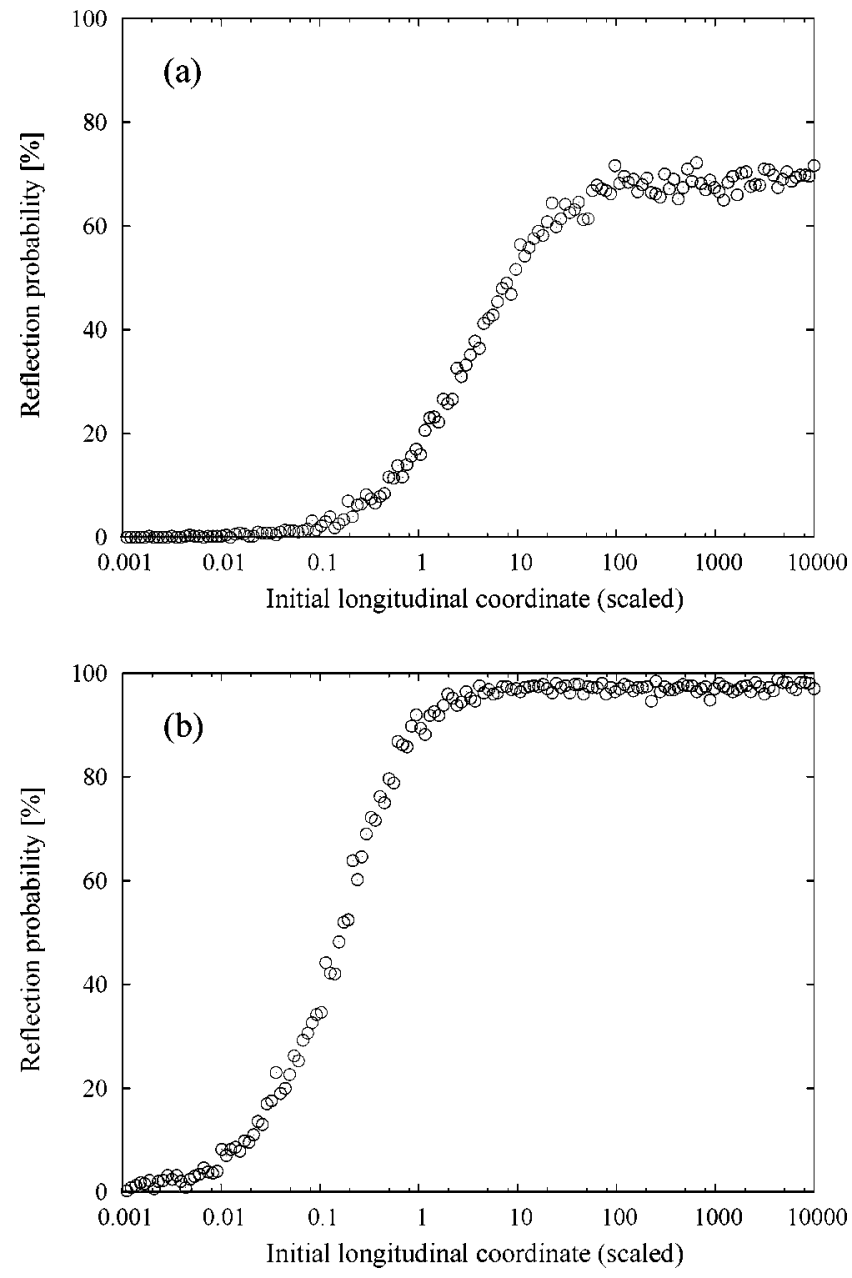

FIG. 1. Dependence of reflection probability on the initial value of $|\hat{z}|$. The initial conditions assumed in these examples are: (a) $\hat{T}_{\perp}=50, \hat{T}_{\|}=0.3$; (b) $\hat{T}_{\perp}=0.1, \hat{T}_{\|}=0.7$.

1000 independent simulations. This figure looks similar to the Hasse's Fig.3 of Ref. [5], except that - due to the different normalization - Hasse's curves are apparently only valid for $\lambda=0.00015$ whereas our Fig. 2 applies for arbitrary density, within the limits outlined above. We recognize that the reflection border is approximately straight, which suggests the ordering condition of the form $\hat{T}_{\perp} \cdot \hat{T}_{\|}^{\alpha}<$ const, where the magnitude of the exponent $\alpha$ is between 2 and 3 . Carrying out the least-square fitting of the $80 \%$ probability curve in Fig. 2, we have the phenomenological ordering criterion

$$
\hat{T}_{\perp} \cdot \hat{T}_{\|}^{2.4}<0.95
$$

which looks satisfactory over the whole temperature range. This simple phenomenological criterion allows us to make a quick estimate of the transition temperature of an ultra-lowdensity beam in a given machine. The criterion (16) is obtained as a fit to the numerical results for the limiting case of a round beam as discussed above. However, we have also studied other aspect ratios $\left\langle\hat{x}^{2}\right\rangle /\left\langle\hat{y}^{2}\right\rangle$, especially the case $\left\langle\hat{y}^{2}\right\rangle \rightarrow 0$. It has been found that the same criterion is a good

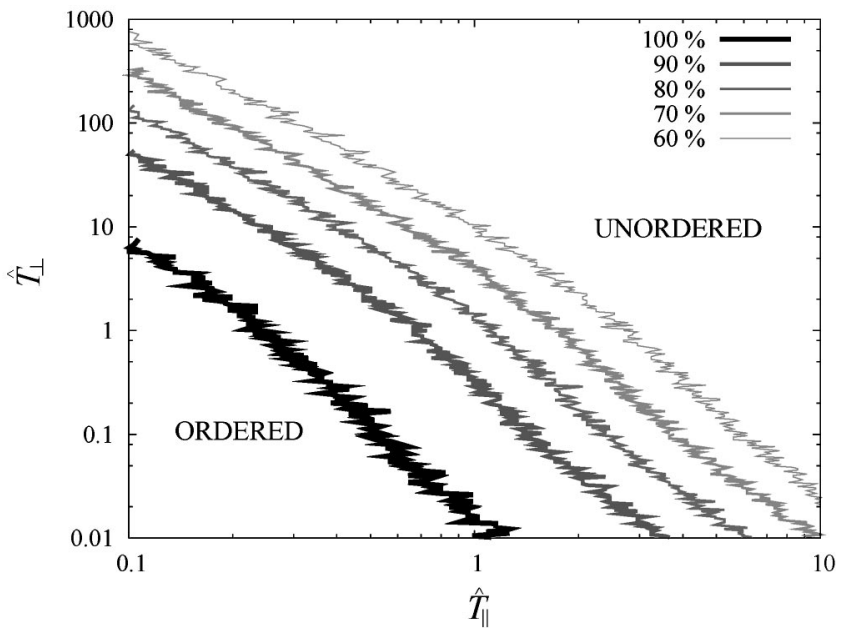

FIG. 2. Reflection probability map obtained numerically from the universal Hamiltonian (14). We have considered a round beam where $\hat{T}_{x}=\hat{T}_{y}$. The initial value of the longitudinal coordinate $\hat{z}$ has been fixed at 100, which is not essential to the result; even if a different value is chosen, we eventually find almost the same map as long as it is sufficiently larger than 10 .

(and, in fact, for high $\hat{T}_{\perp}$ a slightly "pessimistic") approximation even for flat beams.

The actual transition points experimentally confirmed at ESR are plotted on the map in Fig. 3 where the data summarized in Ref. [5] have been used, and are summarized in Table I. (All we need to know here are the values of $\delta p / p$ and $T_{\text {trans }}$ at which anomalous beam behavior was detected.) The scaled average interparticle distance $\tilde{d}$ at transition is also listed in the table. The magnitude of $\tilde{d}$ in each case is on the order of $10^{3}$, which justifies the application of the twoparticle model to the ESR data. We see, from Fig. 3, that the transition points are in-between the $60 \%$ and $80 \%$ contours.

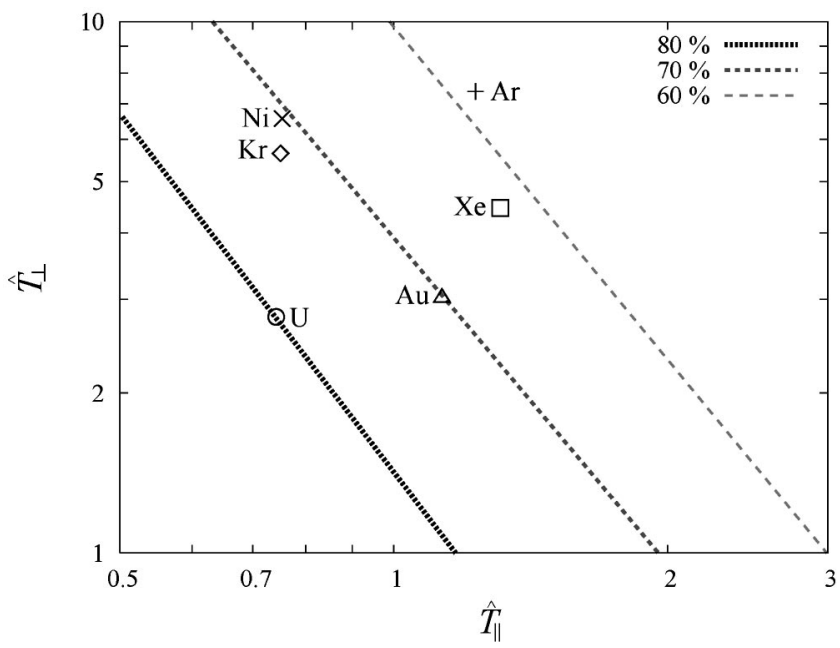

FIG. 3. The operating points at which the anomalous beam behaviors were observed in ESR have been marked with $+\left({ }^{40} \mathrm{Ar}^{18+}\right), \times\left({ }^{58} \mathrm{Ni}^{28+}\right), \diamond\left({ }^{86} \mathrm{Kr}^{36+}\right), \square\left({ }^{132} \mathrm{Xe}^{54+}\right), \Delta\left({ }^{197} \mathrm{Au}^{79+}\right)$, and $\bigcirc\left({ }^{238} \mathrm{U}^{92+}\right)$. The data used here have been summarized in Table I. The three lines show the smoothed probability contours in Fig. 2. 
TABLE I. Momentum spread $\delta p / p$, transverse beam temperature $T_{\text {trans }}$, and scaled interparticle distance $\tilde{d}$ at which anomalous Schottky signals were detected at ESR. The value of $\tilde{d}$ in each case gives the threshold above which the occurrence of $1 \mathrm{D}$ ordering is anticipated. The data below are extracted from the table in Ref. [5].

\begin{tabular}{lcccccc}
\hline \hline & ${ }^{40} \mathrm{Ar}^{18+}$ & ${ }^{58} \mathrm{Ni}^{28+}$ & ${ }^{86} \mathrm{Kr}^{36+}$ & ${ }^{132} \mathrm{Xe}^{54+}$ & ${ }^{197} \mathrm{Au}^{79+}$ & ${ }^{238} \mathrm{U}^{92+}$ \\
\hline Energy $(\mathrm{MeV} / \mathrm{u})$ & 360 & 205 & 240 & 240 & 360 & 360 \\
$\delta p / p\left(10^{-6}\right)$ & 4 & 4 & 4 & 6 & 6 & 5 \\
$T_{\text {trans }}(\mathrm{meV})$ & 300 & 440 & 640 & 1000 & 1500 & 1800 \\
$\tilde{d}$ & 4800 & 5800 & 4600 & 6700 & 1500 & 7400 \\
\hline \hline
\end{tabular}

An example of reflection probability calculated as a function of beam density is given in the Appendix.

Note that the criterion (16) does not predict the final equilibrium temperature after the transition is completed. In fact, according to the ESR data [3], the momentum spread $\delta p / p$ drops at a critical line density by nearly one order of magnitude. After the ordering therefore the marks in Fig. 3 jump to the left where the reflective collision is perfectly guaranteed.

\section{BASIC MECHANISM OF 1D ORDERING}

It is concluded that the universal probability map, Fig. 2, reproduces the ESR data and can be used to analyze a wide range of future experiments and applications. To this end a minimum of information, essentially only the beam temperatures, is necessary. We do not have to be concerned with collective effects, plasma parameters, or density as long as $\tilde{d} \gg 10$. The observed dependence of the transition point on the number of stored ions should not be interpreted as evidence that the line density is of primary importance but rather as an indication that the achievable temperatures depend on beam density.

We next study two-particle Coulomb collisions under the influence of an artificial linear potential in order to derive analytical reflection criteria. The total potential of the system is the sum of two fundamental functions:

$$
V(\hat{x}, \hat{y}, \hat{z})=\frac{1}{2}\left(\hat{x}^{2}+\hat{y}^{2}\right)+\frac{1}{\sqrt{\hat{x}^{2}+\hat{y}^{2}+\hat{z}^{2}}} .
$$

The reflection probability of $100 \%$ implies that the longitudinal coordinate $\hat{z}$ is always bounded in the positive or negative region. When two particles pass each other longitudinally, the value of $\hat{z}$ goes across zero changing its sign. Such an event never takes place if the total energy, which is invariant in the present case, is lower than the potential barrier at $\hat{z}=0$. The function $V(\hat{x}, \hat{y}, \hat{z}=0)$ becomes minimum along $\hat{x}^{2}+\hat{y}^{2}=1$ where the potential height is $3 / 2$. Since the average energy of the system can be expressed as $\hat{E} \approx 2 \hat{T}_{\perp}$ $+\hat{T}_{\|} / 2$, the general criterion of $100 \%$ reflection is given by

$$
2 \hat{T}_{\perp}+\frac{\hat{T}_{\|}}{2}<\frac{3}{2} .
$$

The probability contour corresponding to this condition is, however, rather different from the phenomenological fit in
Eq. (16). Thus the above statistical mechanics argument (space averages over the energy surface is equal to time average over a trajectory) is not applicable. This is attributed to the restriction of the starting conditions; namely, we only wish to consider the particular situation in which two particles are initially located far away from each other and gradually approach. Taking this fact into account, we try to give a plausible explanation to the numerical results in Fig. 2 . For this purpose, it is convenient to look into typical reflection patterns at high and low $\hat{T}_{\perp}$ separately.

\section{A. High transverse temperature: $\hat{T}_{\perp} \gg 1$}

From the Hamiltonian, we find the longitudinal equation of motion $d \hat{p}_{z} / d \theta=d^{2} \hat{z} / d \theta^{2}=\hat{z} /\left(\hat{x}^{2}+\hat{y}^{2}+\hat{z}^{2}\right)^{3 / 2}$, which says that the relative momentum $\left|\hat{p}_{z}\right|$ decreases at a rate proportional to $\left(\hat{x}^{2}+\hat{y}^{2}+\hat{z}^{2}\right)^{-3 / 2}$. It is therefore always possible to let the trajectory go beyond the $\hat{z}=0$ line and, thus have no ordering, by choosing a sufficiently high $\hat{T}_{\perp}$. Inversely speaking, for reflection, $\left|\hat{p}_{z}\right|$ must initially be smaller at higher $\hat{T}_{\perp}$, so that $\hat{p}_{z}$ reaches zero before crossing the $\hat{z}=0$ line.

A large transverse oscillation amplitude also leads to weakening the coupling among the three degrees of freedom. The probability of large energy transfer between the transverse and longitudinal motions will then be lowered. To check this out, a typical trajectory of the two-particle motion is plotted in Fig. 4(a) [14]. We actually see no noticeable change in the oscillation amplitude before and after the reflection, which indicates that the energy of the transverse motion is conserved. By subtracting the transverse energy from both sides of Eq. (14), we obtain the formal equation $\hat{T}_{\|} \approx \hat{p}_{z}^{2}+2 / \sqrt{\sigma_{\perp}^{2}+\hat{z}^{2}}$, where $\sigma_{\perp}$ is the root-mean-squared (rms) amplitude of the transverse oscillation. An approximate ordering condition at high $\hat{T}_{\perp}$ can thus be written as $\hat{T}_{\|}<2 / \sigma_{\perp}$ or, equivalently,

$$
\hat{T}_{\perp} \cdot \hat{T}_{\|}^{2}<2,
$$

because $\sigma_{\perp}^{2}=\left\langle\hat{x}^{2}\right\rangle+\left\langle\hat{y}^{2}\right\rangle=2 \hat{T}_{\perp}$. This condition is similar to the criterion (5) by Meshkov et al., but our reasoning leading to it seems different from the "no-passing" argument of Ref [12]. The reflection border determined by this condition is in reasonable agreement with the numerical result, as shown in Fig. 5. 

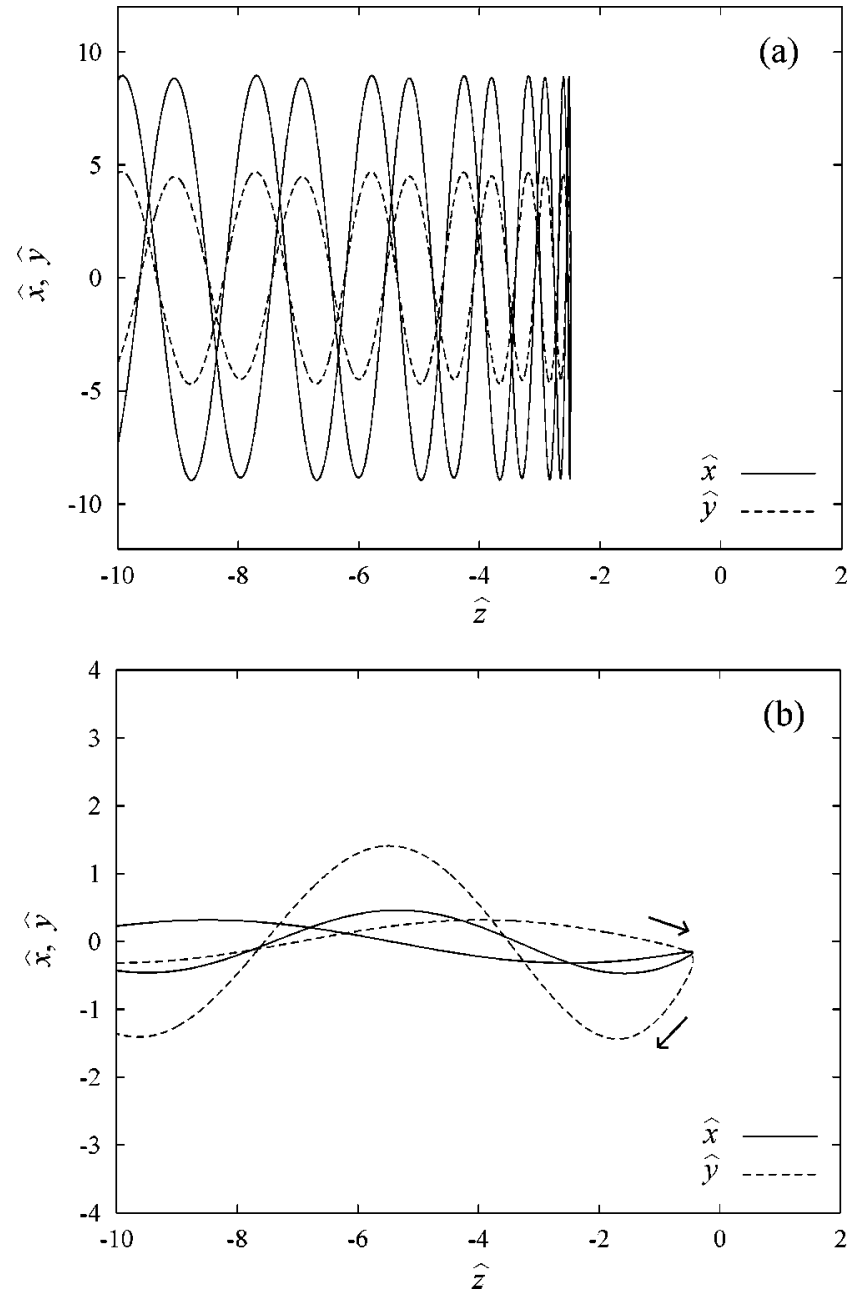

FIG. 4. Typical solutions to the universal equations of motion. The initial coordinates assumed here are: (a) $\left(\hat{x}, \hat{y}, \hat{z} ; \hat{p}_{x}, \hat{p}_{y}, \hat{p}_{z}\right)$ $=(8.944,-3.162,-300 ; 0,3.162,0.5477), \quad$ (b) $\quad\left(\hat{x}, \hat{y}, \hat{z} ; \hat{p}_{x}, \hat{p}_{y}, \hat{p}_{z}\right)$ $=(0,-0.2236,-300 ; 0.3162,-0.2236,2)$. Note that, in the case of high $\hat{T}_{\perp}$ (the upper picture), the horizontal and vertical oscillation amplitudes are independently maintained before and after the reflection.

\section{B. Low transverse temperature: $\hat{T}_{\perp} \ll 1$}

This range is not the one of the present experiments, but it is interesting - for it gives us added insight into the solutions of the two-particle model. The potential function of Eq. (17) produces a steep barrier around the origin. Even high longitudinal temperature is allowed at low $\hat{T}_{\perp}$ and the particle is still reflected. The trajectory can therefore come closer to the origin compared to the high- $\hat{T}_{\perp}$ case. Then, a significant energy transfer from the longitudinal direction to the transverse direction can take place. Figure 4(b) shows a solution to the universal equations of motion, where relatively high longitudinal temperature has been assumed. Naturally, the collision is almost head-on owing to the small transverse oscillation amplitude. We recognize that, in the vicinity of the potential barrier, the collision pattern looks like a Rutherford scattering with a small impact parameter $b$. The scattering angle $\phi$ can thus be estimated from

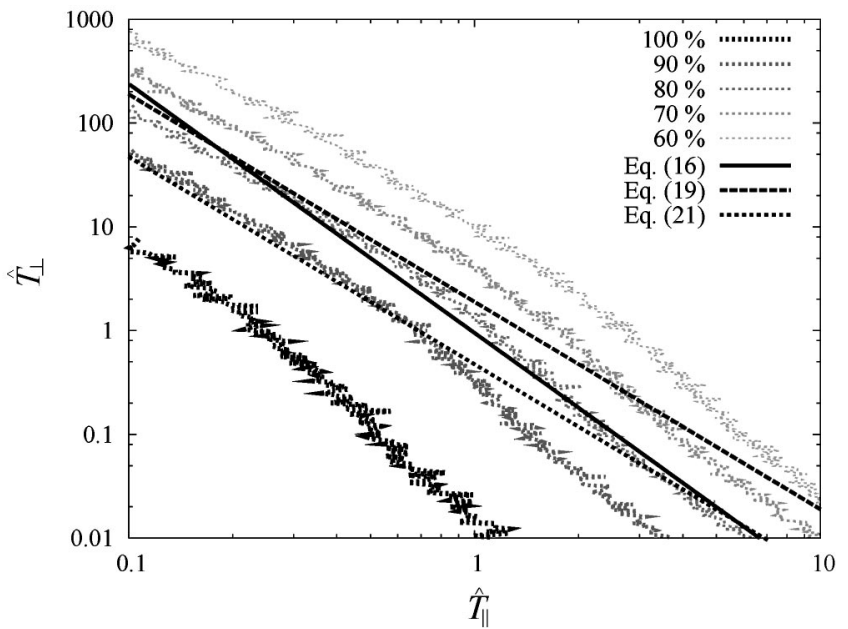

FIG. 5. Reflection probability map. The dotted curves are the probability contours shown in Fig. 2. The thick broken and dotted lines indicate the reflection borders given, respectively, by Eqs. (19) and (21). The phenomenological fit of Eq. (16) has also been plotted in a thick solid line.

$$
\cot \left(\frac{\phi}{2}\right) \approx b \cdot \hat{p}_{\infty}^{2},
$$

where $\hat{p}_{\infty}$ is the total momentum before the scattering. Although the impact parameter depends on the phase and amplitude of the transverse oscillation, we here simply assume $b \approx \sigma_{\perp} \approx \sqrt{2 \hat{T}_{\perp}}$. In addition, $\hat{p}_{\infty} \approx \sqrt{\hat{T}_{\|}}$because $\hat{T}_{\|} \gg \hat{T}_{\perp}$ in the present case. Since $\phi$ must be greater than about $90^{\circ}$ to get a reflection, Eq. (20) results in the ordering condition

$$
\hat{T}_{\perp} \cdot \hat{T}_{\|}^{2}<0.5
$$

In spite of the crude assumptions, this condition qualitatively explains the probability contours in the low $\hat{T}_{\perp}$ region (see Fig. 5). Interestingly, the condition (21) is quite analogous to what we have found for high $\hat{T}_{\perp}$.

\section{SUMMARY}

We have shown that in essence, the ESR-type event is simply determined by Coulomb collisions of two neighboring ions, which under appropriate conditions, can lead to reflections. The Wigner-Seitz radius, the $\Gamma$ parameter, beam density and collective motions are of no substantial importance in this effect.

The two-particle model suffices for explaining the anomalous behavior of electron-cooled ion beams in a storage ring. The dynamical system treated here is made completely parameter free after scaling, which means that the nature of 1D ordering at ultra-low density is only determined by the scaled dimensionless temperatures $\hat{T}_{\perp}$ and $\hat{T}_{\|}$. Details of the lattice design, beam energy, ion species, etc., only enter through the values of $\hat{T}_{\perp}$ and $\hat{T}_{\|}$. Solving the universal equations of motion, we have obtained a useful chart that enables us to estimate the probability of reflective binary collisions at specific beam temperature. An intuitive picture of the ordering pro- 
cess was briefly described to reproduce the reflection border in Fig. 2. The present universal criterion offers experimentalists, who have different storage rings and different types of beams and coolers, a simple guideline on the possible operating region where the sudden transition to the $1 \mathrm{D}$ ordered state is expected.

\section{ACKNOWLEDGMENTS}

Two of the authors (A.M.S. and D.M.) would like to thank Dr. Meshkov for interesting comments. This work was supported in part by the U.S. Department of Energy under Contract No. DE-AC03-76SF0098.

\section{APPENDIX: ESR EXPERIMENTS}

For completeness, let us apply the two-particle algorithm to reproduce the ESR observations. For this purpose, we first need to know the density dependence of the equilibrium beam temperature. The rms momentum spread corresponding to $\delta p / p$ in Table I can be evaluated from the formula $\sqrt{\left\langle(\delta p / p)^{2}\right\rangle}=(8 \ln 2)^{-1 / 2}(\delta p / p)[3]$. The value of $\left\langle(\delta p / p)^{2}\right\rangle$ is then substituted into the definition of $T_{\|}$to find the longitudinal transition temperature. Note, however, that $\delta p / p$ and $T_{\text {trans }}$ in Table I only show their values when a jump of Schottky noise power took place at a specific line density. At a different line density, we must use different $\delta p / p$ and $T_{\text {trans. }}$. According to Ref. [3], the longitudinal and transverse temperature of an electron-cooled beam in ESR scale as $N^{0.6}$ where $N$ is the total number of stored ions. We here employ

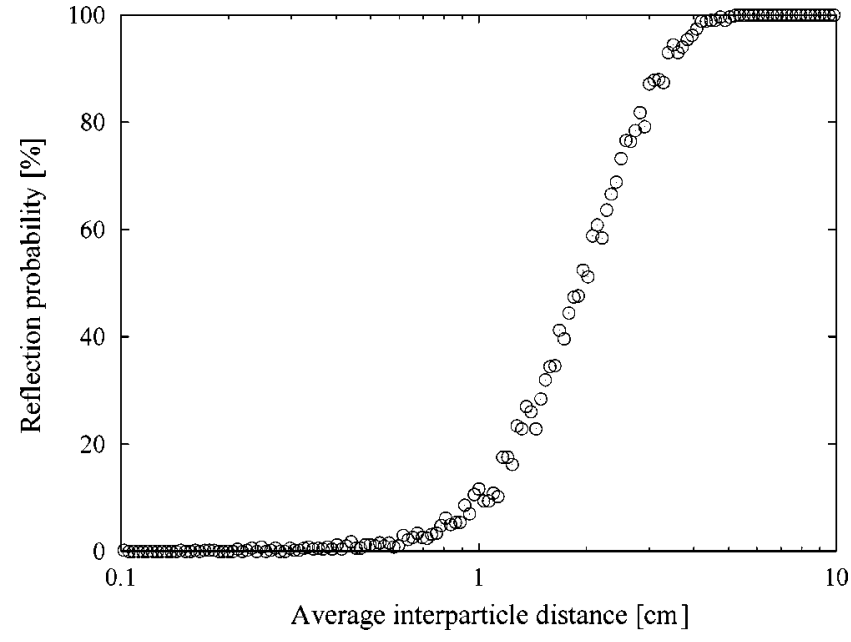

FIG. 6. Two-particle simulation result corresponding to an ESR experiment. A $360-\mathrm{MeV} / \mathrm{u}{ }^{197} \mathrm{Au}^{79+}$ beam in ESR with $\nu_{0}=2.3$ has been assumed. Unlike the numerical procedure outlined in Sec. IV, the initial value of $\left|\hat{p}_{z}\right|$ has been fixed at $\sqrt{\hat{T}_{\|}}$in each simulation.

this scaling law to determine initial conditions for twoparticle simulations. Note also that, as long as the initial value of $\hat{p}_{z}$ is picked from Gaussian random numbers in each simulation run, the reflection probability does not grow as sharply as exhibited in Ref. [5] Therefore, as a trial, we here fix $\hat{p}_{z}$ at $\sqrt{\hat{T}_{\|}}$initially. The reflection probability of ${ }^{197} \mathrm{Au}^{79+}$ ions in ESR then shows the behavior in Fig. 6, which is similar to the results in Ref. [5].
[1] V. V. Parkhomchuk and N. S. Dikansky, Sov. Phys. Tech. Phys. 50, 1411 (1980); E. N. Dementev, N. S. Dikansky, A. S. Medvedko, V. V. Parkhomchuk, and D. V. Pestrikov, Zh. Tekh. Fiz. 50, 1717 (1980); Sov. Phys. Tech. Phys. 25, 1001 (1980).

[2] J. Wei, A. Draeseke, A. M. Sessler, and X.-P. Li, in Proceedings of the Eloisatron Workshop on Crystalline Beams and Related Issues, edited by D. M. Maletic and A. G. Ruggiero (World Scientific, Singapore 1996), p. 229.

[3] M. Steck et al., Phys. Rev. Lett. 77, 3803 (1996).

[4] H. Danared, A. Källberg, K.-G. Rensfelt, and A. Simonsson, Phys. Rev. Lett. 88, 174801 (2002).

[5] R. W. Hasse, Phys. Rev. Lett. 83, 3430 (1999).

[6] R. W. Hasse, Phys. Rev. Lett. 90, 204801 (2003).

[7] J. P. Schiffer and P. Kienle, Z. Phys. A 321, 181 (1985); A. Rahman and J. P. Schiffer, Phys. Rev. Lett. 57, 1133 (1986).

[8] J. Wei, X.-P. Li, and A. M. Sessler, Phys. Rev. Lett. 73, 3089 (1994).

[9] This does not necessarily mean the absence of interparticle correlation within the whole beam. Even if individual particles freely move around for most of the time, they have been longitudinally bounded after the transition, which can give rise to the abrupt change of the Schottky noise power.

[10] The equilibrium temperature of an electron-cooled ion beam is generally determined by the balance between the cooling force and heating due to intrabeam scattering (unless some instability such as coherent resonance is present). As the beam density decreases, the heating rate becomes lower and, accordingly, we can achieve a lower equilibrium temperature. If the ability of an electron cooler is improved (worsened), then the jump of the momentum spread will occur at higher (lower) line density.

[11] Note, however, that the line density of the proton beam in NAP-M was several orders of magnitude higher than that of the heavy ion beams in ESR. As pointed out in Refs. [2,5], the nature of the NAP-M event should be physically different from that of the ESR event.

[12] I. Meshkov, A. Sidorin, A. Smirnov, E. Syresin, and T. Katayama, ISSN 1344-3887, RIKEN-AF-AC-34 (May, 2002); see, also, I. Meshkov, A. Sidorin, A. Smirnov, E. Syresin, T. Katayama, H. Tsutsui, and D. Möhl, Nucl. Instrum. Methods Phys. Res. A (to be published).

[13] $\nu_{x} \approx \nu_{y}$ is actually a reasonable assumption in most cooler storage rings for ion beams.

[14] At high $\hat{T}_{\perp}$, the scattering pattern is quite different from what has been assumed in Ref. [12]. Since $\hat{T}_{\|}$must be low enough for reflection, two countertraveling particles approach very slowly in the longitudinal direction. Consequently, the reflection process advances "gradually" as illustrated in Fig. 4(a). 